\title{
Particle Acceleration in Shock Wave Produced by Coronal Mass Ejection
}

\author{
Kazuyuki Yamashita ${ }^{*}$, Mitsue Den², Tomoya Ogawa ${ }^{3}$, Tatsuo Yoshida ${ }^{4}$ \\ 1 University of Yamanashi, Kofu, Yamanashi, Japan. \\ 2 National Institute of Information and Communications Technology, Tokyo, Japan. \\ ${ }^{3}$ Kitasato University, Kanagawa, Japan. \\ ${ }^{4}$ Ibaraki University, Mito, Ibaraki, Japan. \\ * Corresponding author. Tel.: +81-55-220-8160; email: kazuyuki@yamanashi.ac.jp \\ Manuscript submitted October 13, 2014; accepted December 27, 2014. \\ doi: 10.17706/ijapm.2015.5.1.8-17
}

\begin{abstract}
The energetic particle event that occurred on 314 DOY in 2000 is a good sample to investigate particle acceleration mechanisms because both the coronal mass ejection and the energetic particles were observed. We obtained energetic particles using the stochastic differential equation method with the velocity of the shock wave and the assumptions of the energy spectrum of the input pre-accelerated particles and the diffusion coefficient. The behavior of the shock wave produced at a point on the solar surface was obtained by hydrodynamic simulation. The simulation was carried out by the adaptive mesh refinement scheme in three-dimensional space. We tested the models of the diffusion coefficient. We concluded that the spectra at the time when the shock arrived at $1 \mathrm{AU}$ agreed with the observed data but those at earlier times were small, and that the non-constant diffusion coefficient model, which gives an increase with the energy of the particles, cannot explain the low-energy component in the observed spectra. Therefore, the models of the pre-accelerated ambient particles and the diffusion coefficients should be improved.
\end{abstract}

Key words: Adaptive mesh refinement, coronal mass ejection, shock wave, stochastic differential equation.

\section{Introduction}

It is important to predict energetic particle events because the energetic particles in space sometimes damage devices installed in satellites. If such events are followed by events on the solar surface, such as $\mathrm{X}$-ray flares, it may be possible to avoid fatal effects in these devices because there is sufficient time to move the satellite out of the path after detecting the solar surface events. It is generally accepted that particles are accelerated in the first Fermi acceleration mechanism associated with the interplanetary shock wave formed by coronal mass ejections (CMEs). There are many variances in the observed time-intensity profiles and particle spectra at $1 \mathrm{AU}$, which are considered to be due to a number of factors. Den et al. [1] classified the energetic particle events into four categories according to the maximum energy of the accelerated particles, and the time scales of precursor and of being enhancing the particle flux. Den et al. [2] modeled the typical so called Type 2 event that occurred on 314 DOY (Nov. 8) in 2000. The category of Type 2 class events is as follows: source CMEs are accompanied by large X-ray flares so that the solar energetic particles and the shock accelerated particles are thought to be mixed. The associated shock waves are strong and the maximum energy of accelerated particles is $>10 \mathrm{MeV}$. The study of Den et al. [2], examined whether the CME-associated shock wave can accelerate particles to energies $>10 \mathrm{MeV}$. They focused on the energy of 
seed populations of particles and concluded that injection of high-energy particles was necessary for early enhancement of high-energy proton flux. With regard to "mixed particles," Cane et al. [3] studied 29 solar particle events and found four events in which both characters of a Fe/O ratio for impulsive events and for gradual SEP events existed and exhibited double peaks in the intensity profile. They concluded that there were two components in major solar particle events, which was consistent with the results of Den et al. [2]. $\mathrm{Li}$ and Zank [4] reproduced a "two-peak" feature in their simulations naturally by inputting particles corresponding to those accelerated in a flare process by using the sophisticated method of Zank et al. [5] and Rice et al. [6], which included modeling of the evolution and propagation of the CME-driven shock using an MHD simulation and of particle acceleration using an onion shell-like model that could be applied to shocks of arbitrary strength. Their simulation results agreed with those of Den et al. [2].

In this paper, we extend the work of Den et al. [2] to study the same event that occurred on 314 DOY (Nov. 8) in 2000. They solved the governing equations of the evolution of the trapped particles using the stochastic differential equation (SDE) method, which was useful compared to solving the Fokker-Planck equation, and did not assume the shell structure for the shock. They assumed that the diffusion coefficient was constant as the Bohm form and with a hand-made simple velocity field. However, the model was unrealistic in evaluating the velocity field and possibly in taking the diffusion coefficient to be constant. They used constant downstream velocities with the shock front velocity observed at 1AU.

A realistic velocity field can be obtained by hydrodynamic simulation. However, it is necessary to perform the simulation with high spatial resolution because the spatial differentiation of the velocity is the essential part in accelerating particles. High resolution is achieved by using a large number of cells but the computation requires a great deal of CPU time. The adaptive mesh refinement (AMR) scheme applied to the hydrodynamic solver achieves high spatial resolution around the regions where the hydrodynamic variables change markedly in space and it minimizes the number of cells required because it allows the cells to be fine only around such regions. One of the AMR schemes was proposed by Khokhlov [7] and it was combined with the monotone upwind scheme for conservation laws (MUSCL) of the Roe method by Ogawa et al. [8]. The MUSCL is a scheme with the total variation diminishing (TVD) property andhigh spatial accuracy. The Roe method [9] can capture a shock within a small number of cells. Ogawa et al. [8] found that the CME-driven shock was captured with high resolution.

This article consists of the following sections: Section II describes the fundamental methods used to obtain the spectra of the accelerated particles; Section III describes the models adopted in the simulations; Section IV presents the results; and Section V gives the concluding remarks.

\section{Methods}

\subsection{Hydrodynamic Simulation}

We obtain the spectrum of the accelerated particles in two steps. First, the velocity field is obtained by the hydrodynamic simulation. Second, the energy spectrum of the particles is obtained by the stochastic differential equation (SDE) method.

To obtain the interplanetary velocity field, we carry out 3-dimensional hydrodynamic simulations using the code developed by Ogawa et al. [8]. The code consists of the MUSCL-Roe method of 3rd order in space with the AMR scheme of Khokhlov [7], where the computational cells are refined/destroyed by checking that there exists a shock around the cell.

The hydrodynamic state at the time before the shock wave is produced should be the steady state of the interplanetary solar wind environment. This state is obtained by taking 13 days after the simulation starts and then, at $t=0$, the kinetic energy, $1 \times 10^{32} \mathrm{erg}$, is input at the solar surface, which represents the coronal mass ejection event. 
In the simulation, the heat ratio is, the side length $L$ of the simulation box is $L=4.65 \mathrm{AU}$, the side length of the coarsest cell is $L / 128$, the side length of the finest cell is $L / 16384$, the sun is located at the center of the box, and the time step is determined by the stability condition. The simulation is ended at time $t=4$.5days after the shock wave starts.

\subsection{Stochastic Differential Equations Method}

We use the stochastic differential equations method coupled with particle splitting to obtain the spectrum of the accelerated particles, as formulated by Yoshida and Yanagida [10].

The Fokker-Planck form of the cosmicray transport equation in the spherically symmetric geometry assumed is given by

$$
\frac{\partial \varphi}{\partial t}=-\frac{\partial}{\partial r}\left\{\left(v_{r}+\frac{2 \kappa}{r}\right) \varphi\right\}+\frac{\partial^{2}}{\partial r^{2}}(\kappa \varphi)+\frac{\partial}{\partial u}\left\{\frac{1}{3}\left(\frac{\partial v_{r}}{\partial r}+\frac{2 v_{r}}{r}\right) \varphi\right\}
$$

with

$$
u=\log \left(\frac{p}{m_{p} c}\right)
$$

and

$$
\varphi=4 \pi r^{2} p^{3} f
$$

where $t$ is the time, $u$ is the radial solar wind speed, $p$ is the proton momentum, $f$ is the distribution function for protons, $v_{r}$ is the radial velocity field, $\kappa$ is the spatial diffusion coefficient, $m_{p}$ is the proton mass, and $c$ is the speed of light.

The stochastic differential equations equivalent to Eq. (1) are

$$
d r=\left(v_{r}+\frac{2 \kappa}{r}\right) d t+\sqrt{2 \kappa} d W_{r}
$$

And

$$
d u=-\frac{1}{3}\left(\frac{\partial v_{r}}{\partial r}+\frac{2 v_{r}}{r}\right) d t
$$

where $d W_{r}$ is a Wiener process so that the probability of taking $d W_{r}$ is given by the Gaussian form as

$$
P\left(d W_{r}\right)=\frac{1}{\sqrt{2 \pi d t}} \exp \left(-\frac{d W_{r}^{2}}{2 d t}\right) .
$$

Time integration is performed by a simple Euler method. We use the number of pre-accelerated particles, $n=10000$, and the time step $d t=22.13 \mathrm{~s}$, which satisfies the conditions regarding diffusion and advection 
reported by Külls and Achterberg [11].

To minimize the statistical fluctuation, the number of particles should be large. However it is inconvenient to prepare large numbers of pre-accelerated particles because the CPU time is wasteful for computing the particles that are not accelerated. Thus, the particle splitting is performed so that a particle in which the energy exceeds the threshold vvalues divides into two particles each of which has half the statistical weight. The threshold energies $E_{i}$ for $i=1,2, \ldots, 10$ are here taken to be $E_{i+1} / E_{i}=10^{1 / 10}$ and $E_{10}=0.5 \mathrm{MeV}$.

The radial velocity $v_{r}$ and its radial gradient $\partial v_{r} / \partial r$ at the position of the particle are evaluated by interpolating the velocities at the neighboring cells and at the two nearest time steps, which are obtained by the hydrodynamic simulation.

\section{Models}

We model the diffusion coefficient and the input particles as follows.

\subsection{Diffusion Coefficient}

The diffusion coefficient is simply considered to be a constant value, $2.7 \times 10^{19} \mathrm{~cm}^{2} / \mathrm{s}$, which is denoted as model KC. This value was obtained by Den et al. [2] as a result of fitting the spectra for models of the constant diffusion coefficients, formulated by Blandford and Ostriker [12], to the observed spectrum of the CME-driven shock that occurred on 314 DOY 2000. Here, we adopt the model in which the diffusion coefficient varies as proposed by Zank et al. [5] as a counterpart. In this model, the diffusion coefficient depends on the energy of the particle and the ambient magnetic field as follows:

$$
\kappa(p, B)=\frac{\kappa_{0}}{A(k)} \frac{B_{0}}{B} \frac{p / p_{0}}{\sqrt{\left(m_{p} c / p_{0}\right)^{2}+\left(p / p_{0}\right)^{2}}}
$$

with

$$
\kappa_{0}=\frac{4}{3 \pi} \frac{p_{0} c}{e B_{0}}
$$

where $B$ is the intensity of the ambient magnetic field, $A(k)$ is the wave number density per unit logarithmic bandwidth in wave number $k, p_{0}$ is the constant momentum scale, and $e$ is the electron charge. To obtain the diffusion coefficient at the shock front where the strong shock limit is imposed, according to Zank et al. [5], we use $A(k)=1$. The model given by Eqs. (7) and (8) with $A(k)=1$ is denoted as model KZ. The intensity of the ambient magnetic field $B$ is given by the following formula according to Parker[13]:

$$
B=B_{0}\left(\frac{R_{0}}{r}\right)^{2}\left\{1+\left(\frac{\Omega_{0} R_{0}}{u}\right)^{2}\left(\frac{r}{R_{0}}-1\right)^{2} \sin ^{2} \theta\right\}^{1 / 2},
$$

where $\theta$ is the co-latitude of the solar wind with respect to the solar rotation axis, $\Omega_{0}$ is the solar rotation rate, and $B_{0}$ is the intensity of the magnetic field at the co-rotation radius $R_{0}$. We use $\theta=90^{\circ}, R_{0}=10 R_{S}$, $B_{0}=1.83 \times 10^{-6} \mathrm{~T}, u=400 \mathrm{~km} / \mathrm{s}$, and $\Omega_{0}=2 \pi /(25.4$ days $)$. The diffusion coefficient for model $\mathrm{KZ}$ is shown in Fig. 1. The coefficient increases almost linearly with the energy of the particle. 


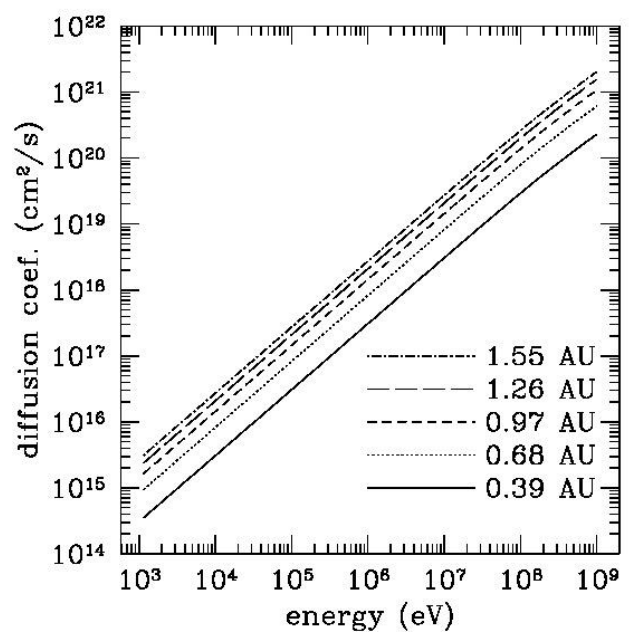

Fig. 1. The diffusion coefficient, $k$ for model KZ.

\subsection{Input Particles}

The energy spectrum of the ambient particles is simply assumed to be the observed pre-shocked spectrum.

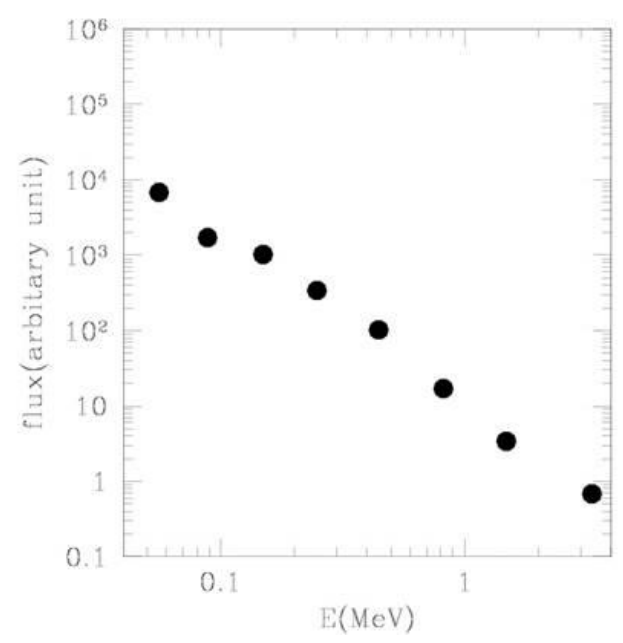

Fig. 2. The energy spectrum for the input particles.

This is taken from the data observed by ACE before CME occurred on 314 DOY 2000.

The ambient pre-accelerated particles are input at the shock front at each time step.

\section{Results}

\subsection{Velocity Field}

The radial interplanetary velocity fields, $v_{r}(x, y)$, on the equatorial plane are shown in Fig. 3.The coordinate is co-rotated with the motion of the earth around the sun. While the shock starts in the direction of N00W45, it arrives at the region in this direction because the time scale of shock propagation is much shorter than that of the motion of the earth. The spread angle of the shock is kept small and thus the energy of the shock does not disperse much.

Acceleration is most effective for the direction in which the velocity gradient is maximum and this is the radial direction as shown in Fig. 3. Therefore, we use the radial velocity to calculate acceleration according 
to Eqs. (4)-(6).

The radial velocities, $v_{r}(r)$, along the line of the shock propagation direction at several time points are shown in Fig. 4. As shown in the figure, the acceleration of the particles effectively takes place at the shock front as the radial gradient of the radial velocity, $\partial v_{r} / \partial r$, which is the essential part of the particle energy gain seen in Eq. (5), is maximized in the absolute values at the shock front and that the shock front velocity decreases over time, which gives a larger radial gradient in the earlier phase.

The shock front that gives the most effective point in particle acceleration is detected by searching the maximum point of the radial gradient of $v_{r}$.

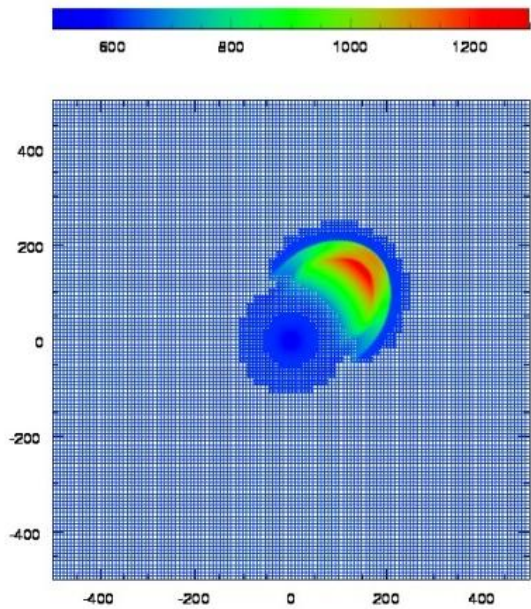

Fig. 3. The radial velocity field on the equatorial plane at 1.39 days after the shock starts.

The sun is located at $(0,0)$ and the earth orbit is $r=224$ in this coordinate. The mesh lines show the meshes used in the AMR simulation. The colors present the radial velocities $v_{r}$ corresponding to the color bar; for example, red indicates $v_{r}>1200 \mathrm{~km} / \mathrm{s}$.
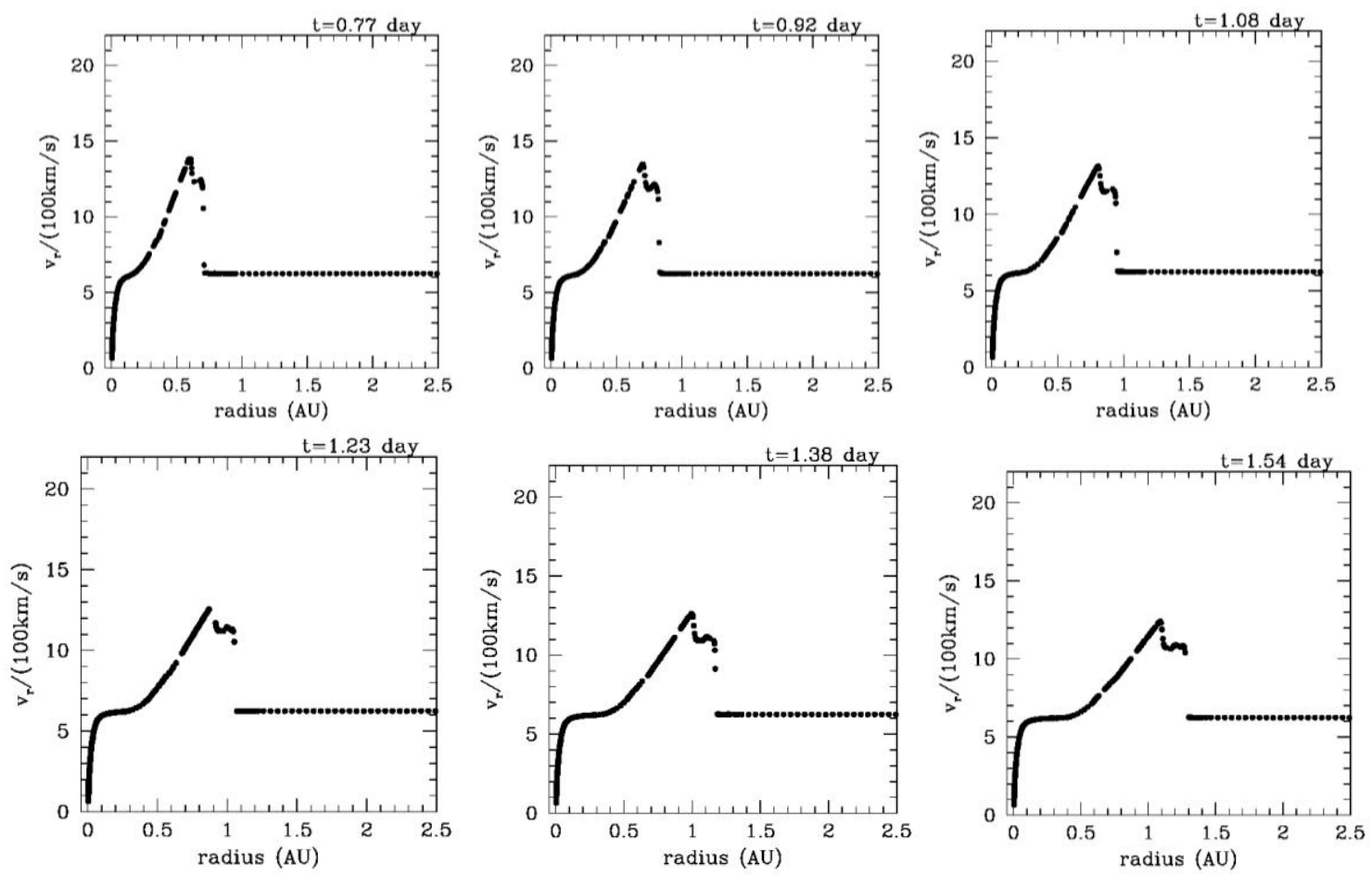

Fig. 4. The radial velocity field along the line of the shock propagation direction on the equatorial plane. 
The times $t=0.77,0.92,1.08,1.23,1.38$, and 1.54 days are shown.

\subsection{Diffusion Coefficient}

To see the diffusion coefficients in our calculation, we plot the diffusion coefficient for every particle at every time step in model KZ, as shown in Fig. 5.

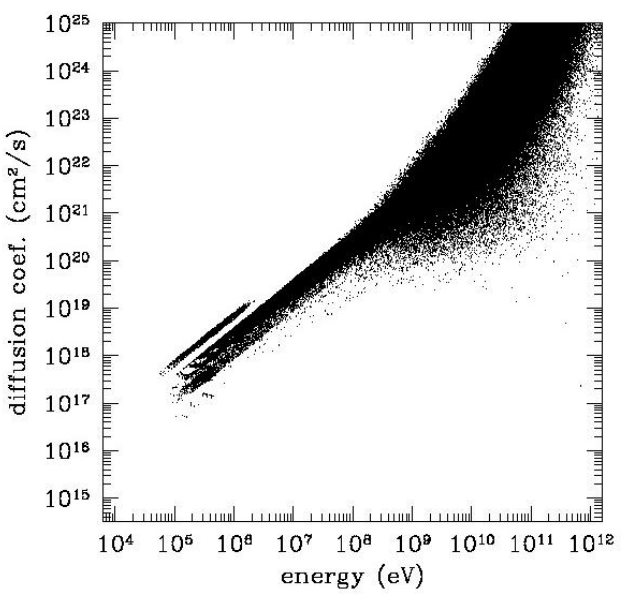

Fig. 5. Diffusion coefficients for model KZ. The energy of the particle and the diffusion coefficient are plotted.

The diffusion coefficients in model $\mathrm{KZ}$ are proportion to the energy of the particle, which is consistent with Fig. 1. We note that the diffusion coefficients in model $\mathrm{KZ}$ are smaller for energies below $10 \mathrm{MeV}$ and larger for energies above $10 \mathrm{MeV}$ than the constant value $\kappa=2.7 \times 10^{19} \mathrm{~cm}^{2} / \mathrm{s}$ in model $\mathrm{KC}$.

\subsection{Spectra of the Accelerated Particles}

After the CME on 314 DOY 2000, the spectra of the particles are observed as shown in Fig. 6.

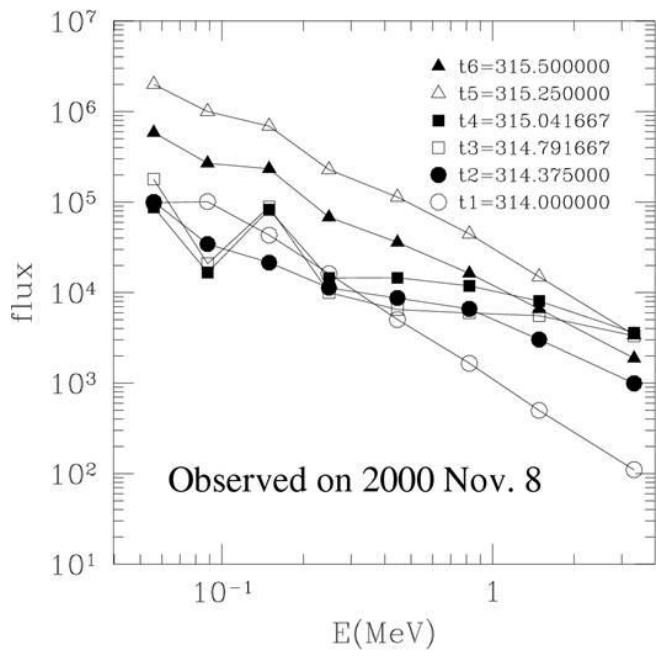

Fig. 6. The spectra after the CME on 314 DOY 2000.The shock arrived at $1 \mathrm{AU}$ at $\mathrm{t}_{5}$.

The shock passed through $1 \mathrm{AU}$ at $\mathrm{t}_{5}$ and at this time the spectrum is compared to thecalculated spectra. The spectra of the accelerated particles at $r=0.9-1.1$ AU are shown in Fig. 7.

Panel (a) shows the spectra for model KC, in which the diffusion coefficient is constant. The spectra increase within 1.23 days and gradually decrease maintaining the power indices although they are harder 
than the observations. Panel (b) shows the spectra for model KZ, in which the diffusion coefficient varies. The power indices at 1.23 and 1.38 days for model KZ reproduce the observed values better than for model $\mathrm{KC}$. This is because the high-energy components for model $\mathrm{KZ}$ become smaller than those for model $\mathrm{KC}$ as the diffusion coefficients for energies above $10 \mathrm{MeV}$ are larger for model $\mathrm{KZ}$ than for model $\mathrm{KC}$ and thus the energetic particles escape away from $1 \mathrm{AU}$ in the early phase. However, the components below $10 \mathrm{MeV}$ drop markedly at 1.54 days for model KZ, and this result does not agree with the observations. This is because the diffusion coefficients for energies below $10 \mathrm{MeV}$ are smaller for model $\mathrm{KZ}$ than for model $\mathrm{KC}$. The flux is commonly maximized at the time point when the shock passes through $1 \mathrm{AU}$ and is small before 1.08 days.

Comparing the results with those of the toy model shown by Den et al. [2], the spectra are found to be harder and the acceleration is more effective as the absolute value of the velocity gradient in the early phase becomes larger.

The spectra at $r=0.7-0.9 \mathrm{AU}$ are shown in Fig. 8 .

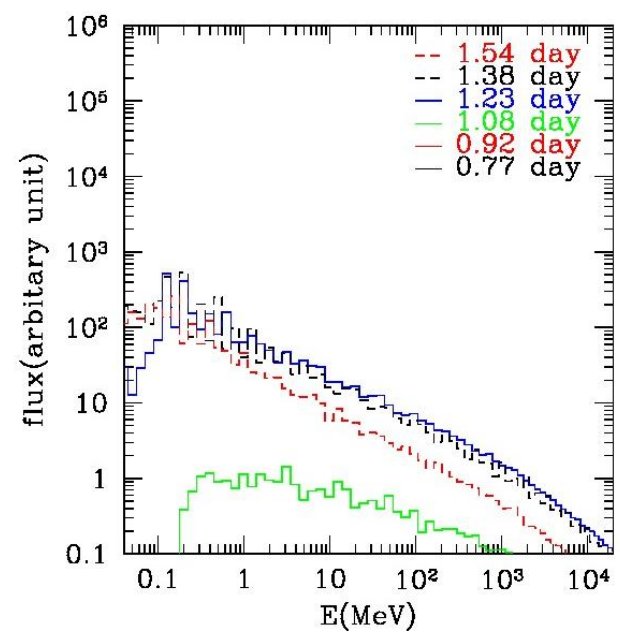

(a)

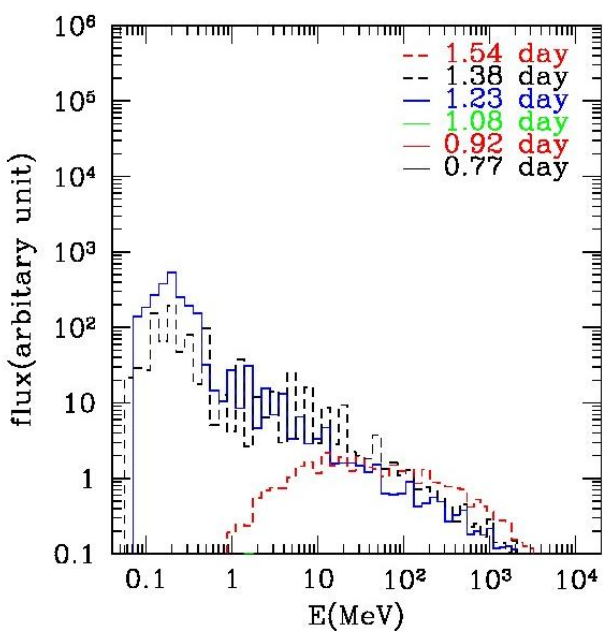

(b)

Fig. 7. The spectra of the particles in the region between $r=0.9-1.1 \mathrm{AU}$ at time $0.77,0.93,1.08,1.23,1.39$, and 1.54 days after the shock wave starts on the solar surface; (a) for model KC and (b) for model KZ. The CME-driven shock passes through $1 \mathrm{AU}$ at 1.23 days.

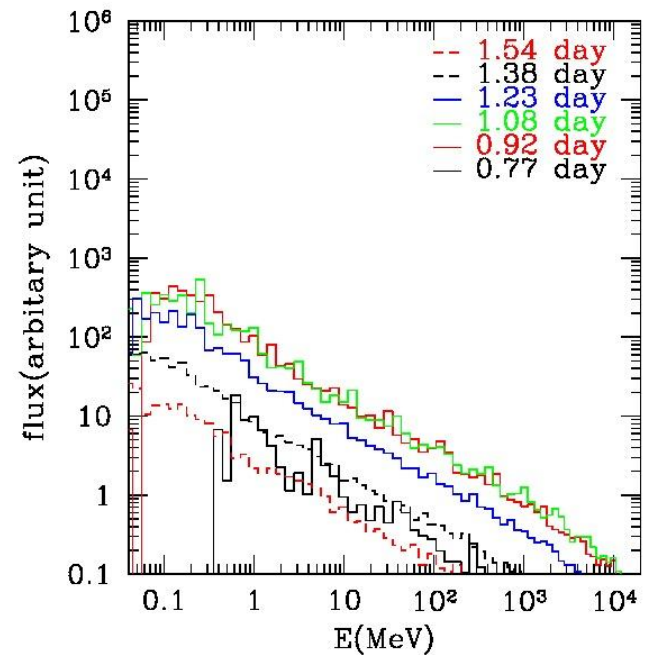

(a)

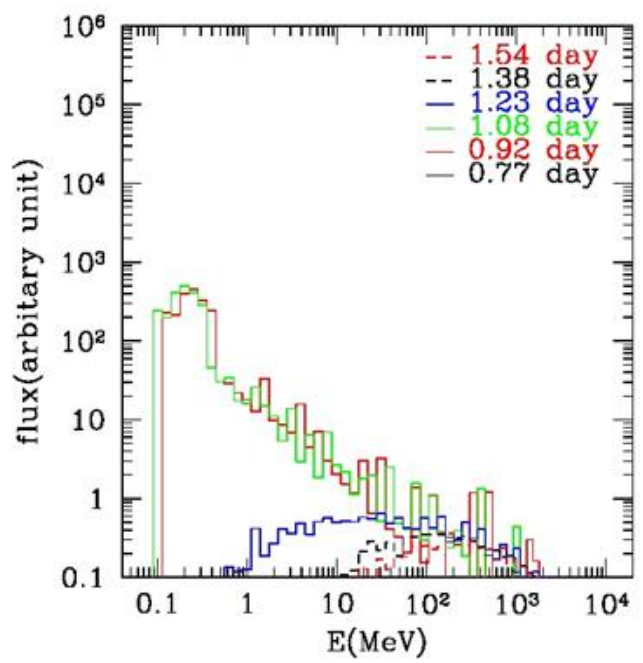

(b)

Fig. 8. The spectra at 0.7-0.9AU; (a) for model KC and (b) for model KZ. 
The flux is maximized around the time 0.92 days when the shock arrives at the region. When the region becomes downstream of the shock, the fluxes decrease and the damping rate in the lower energies is larger for model KZ than for model KC. This behavior is similar to that at 0.9-1.1 AU at 0.77 days. There exist particles with energies higher than $10 \mathrm{MeV}$. This component is explained by the reflection of energetic particles back to this region by diffusion.

\section{Concluding Remarks}

We investigated particle acceleration in the CME-driven shock using the AMR hydrodynamic3D simulation to obtain the velocity field and the SDE method to calculate the energies and locations of the particles. This method is useful and has been established because the models can be tested under realistic conditions and the obtained results can be explained physically.

The velocity field obtained by the hydrodynamic simulation shows the downstream velocities to be larger in the earlier phase and the acceleration is more effective than that described by Den et al. [2]. Model KZ in which the diffusion coefficient increases with the energy of the particles shows a deficit in the low-energy particles after the shock passes. Therefore, it is necessary that the diffusion coefficients below $10 \mathrm{MeV}$ are larger than the values adopted in model KZ. The result indicating that the flux is small before 1.08 days does not agree with the observations, suggesting that the ambient particles already have larger energies before the particles meet the shock, as assumed by Den et al. [2].

\section{Acknowledgment}

Hydrodynamic simulation in the study was performed with a high performance computer system at National Institute of Information and Communication Technology (NICT) as a collaborative research project.

\section{References}

[1] Den, M., Yoshida, T., \& Yamashita, K. (2001). Particle acceleration in interplanetary shocks: Classification of energetic particle events and modeling. Solar and Galactic Composition, 598, 323-328.

[2] Den, M., Yoshida, T., \& Yamashita, K. (2003). Modeling of "gradual" solar energetic particle events using a stochastic differential equation method. Advances in Space Research, 32(12), 2685-2690.

[3] Cane, H. V., Rosenvinge, T. T., Cohen, C. M. S., \& Mewaldt, R. A. (2003). Two components in major solar particle events. Geophysical Research Letters, 30(12), SEP 5-1, 8017.

[4] Li, G., \& Zank, G. P. (2005). Mixed particle acceleration at CME-driven shocks and flares. Geophysical Research Letters, 32(2), L02101.

[5] Zank, G. P., Rice, W. K. M., \& Wu, C. C. (2000). Particle acceleration and coronal mass ejection driven shocks: A theoretical model. Journal of Geophysical Research, 105(A11), 25079-25096.

[6] Rice, W. K. M., Zank, G. P., \& Li, G. (2003). Particle acceleration at coronal mass ejection driven shocks: For arbitrary shock strength. Journal of Geophysical Research, 108(A10), SSH 5-1, 1369.

[7] Khokhlov, A. M. (1998). Fully threaded tree algorithms for adaptive refinement fluid dynamics simulations. Journal of Computational Physics, 143(2), 519-543.

[8] Ogawa, T., Den, M., Tanaka, T. \& Yamashita, K. (2006). Simulation of interplanetary shock wave caused by CME on August 25, 2001. Advances in Geosciences, 2, 65-71.

[9] Roe, P. L. (1981). Approximate Riemann solvers, parameter vectors, and differential schemes. Journal of Computational Physics, 43, 357-372.

[10] Yoshida, T., \& Yanagita, S. (1994). A stochastic simulation method for particle acceleration and non-thermal photon emission in astrophysical processes. Progress of Theoretical Physics, 92(6), 
1217-1222.

[11] Külls, W. M., \& Achterberg, A. (1994). Computation of cosmic-ray acceleration by Ito's stochastic differential equations. Astronomy and Astrophysics, 286, 314-327.

[12] Blandford, R. D., \& Ostriker, J. P. (1978). Particle acceleration by astrophysical shocks. Astrophysical Journal, Part 2 - Letters to the Editor, 221, L29-L32.

[13] Parker, E. N. (1958). Dynamics of the interplanetary gas and magnetic fields. Astrophysical Journal, 128, 664-676.

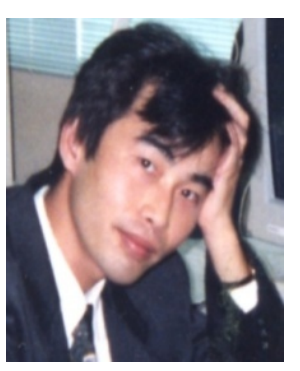

Kazuyuki Yamashita was born in Kagawa, Japan. He was educated at the Dept. of Phys. of Kyoto Univ., Japan and earned a doctorate in physics at this university in 1993. His major field of study is astrophysics.

He worked at Chiba University in Japan from 1993 to 2003, and since 2003, he has worked at the University of Yamanashi, Kofu, Japan. He has published many articles, such as, "Clustering effect on the number count of faint galaxies," in Progress of Theoretical Physics, vol. 88, no. 2, pp.283-289, "A numerical study of galaxy formation and the large scale structure of the universe," in Progress of Theoretical Physics, vol. 89, no. 2, pp. 355-369, and "Simulation of interplanetary shock wave caused by CME on August 25 2001," in Advances in Geosciences, vol. 2: Solar Terrestrial, pp. 64-71. His previous research interests are galaxy formation, mixing in supernova envelopes, and hydrodynamic simulation method. His current interest is particle acceleration in space plasma.

Dr. Yamashita is a member of the Astronomical Society of Japan. 